\title{
Molecular Dynamics Study of 2-Nitrophenyl Octyl Ether and Nitrobenzene
}

\author{
Miguel Jorge, ${ }^{* \dagger}$ Rubin Gulaboski, ${ }^{\dagger,+, \S}$ Carlos M. Pereira, $;$ and M. Natália D. S. Cordeiro $*, \dagger$ \\ REQUIMTE and CIQ-UP L4, Faculdade de Ciências, Universidade do Porto, Rua do Campo Alegre 687, \\ 4169-007 Porto, Portugal
}

Received: March 1, 2006; In Final Form: April 7, 2006

\begin{abstract}
The pure organic liquids nitrobenzene (NB) and 2-nitrophenyl octyl ether (NPOE) have been studied by means of molecular dynamics simulations. Both solvents are extremely important in various interfacial processes, mainly connected with ion transfer taking place across the interface with water. Thermodynamic (mass density, enthalpy of vaporization, isothermal compressibility, dipole moment) and dynamic (viscosities and self-diffusion coefficients) properties of both liquids have been calculated and are in very good agreement with the experimental data. In the case of NB, several potentials have been tested and the obtained results compared and discussed. In most cases, the OPLS all-atom potential gives results that are in better agreement with available experimental values. Atomic radial distribution functions, dihedral and angle distributions, as well as dipole-orientation correlation functions are used to probe the structure and interactions of the bulk molecules of both organic solvents. These were seen to be very similar in terms of structure and thermodynamics, but quite distinct in terms of dynamic behavior, with NPOE showing a much slower dynamic response than NB. A simulation study of the simple $\mathrm{Cl}^{-}$and $\mathrm{K}^{+}$ions dissolved in both solvents has been also undertaken, revealing details about the diffusion and solvation mechanisms of these ions. It was found that in both liquids the positive potassium ion is solvated by the negative end of the molecular dipole, whereas the negative chloride ion is solvated by the positive end of the dipole.
\end{abstract}

\section{Introduction}

Considering that many processes take place across the interface of two conjoined immiscible liquids, an appropriate and suitable selection of the organic solvents is, indeed, of crucial importance for their efficiency. The accomplishment of various interfacial phenomena, such as extraction, interfacial catalysis, thermodynamics and kinetics of ion and electron transfer processes across the liquid|liquid interfaces, depends significantly on the features of the organic solvent used. As a rule, an ideal organic solvent for the above-mentioned interfacial processes should be stable, have low water-miscibility, relatively low vapor pressure and no (or, at least, no significant) harmful effect on humans. Among the various organic solvents employed for studying the interfacial processes so far, nitrobenzene (NB), 1,2-dichloroethane, and $n$-octanol are the most widely used. ${ }^{1}$ The former two have been very prominent in studies of ion transfer across the interface between two immiscible liquids. ${ }^{1}$ Although nitrobenzene has a low miscibility with water, it is a good medium for solvation of ions, due to its high dielectric constant of 35.6.2,3 However, its widespread usage for both research and industrial purposes is limited by its high toxicity and carcinogenic effects. ${ }^{3}$ In the past decade, 2-nitrophenyl $n$-octyl ether (NPOE) has emerged as a viable alternative, and it is seen as one of the most promising solvents in ion transfer studies across liquid|liquid interfaces. ${ }^{2,4-12}$ Compared to the other organic solvents, NPOE has a relatively low solubility in water, a low vapor pressure, and is not known to be highly

* Corresponding authors. E-mail: miguel.jorge@fc.up.pt (M.J.); ncordeir@fc.up.pt (M.N.D.S.C.).

$\dagger$ REQUIMTE.

$\div$ CIQ-UP L4.

$\S$ On leave from the Department of Chemistry, Faculty of Natural Sciences and Mathematics, Sts. Cyril and Metodij University, Skopje, Macedonia. toxic. ${ }^{3}$ In addition, NPOE has a high molar volume and a medium permittivity $\left(\epsilon_{\text {rel }}=24.2\right)$, which makes it also a good solvent for ionic solutes. Furthermore, its molecular structure is interesting for the investigation of the effect of properties and structure of the solvent molecules on the thermodynamics and kinetics of ion transfer. ${ }^{2}$ NPOE is very suitable for designing potentiometric or voltammetric electrochemical sensors for ionic or ionisable species. It has been applied in pharmacology, ${ }^{2,7,12}$ and in the design of voltammetric and amperometric sensors and detector devices based on ion transfer. ${ }^{6,8-10}$ It is also worth mentioning that a recent study has shown that the solvation properties of nitrobenzene and NPOE toward ionic species are very similar. ${ }^{11,12}$

In contrast, few theoretical studies have been devoted to probe such organic solvents. NB has been studied by molecular simulation in the context of light scattering, ${ }^{13}$ and of liquid|liquid interfaces, ${ }^{14-16}$ as well as for the optimization of force-field parameters. ${ }^{17,18}$ Although some useful information can be extracted from those studies, none has presented a systematic and detailed analysis of the thermodynamic and dynamic properties of the bulk liquid. As for NPOE, there have been, to the best of our knowledge, no previous simulation studies of this liquid. The present work intends to fill that gap by reporting the results obtained from molecular dynamics (MD) simulations of pure NB and NPOE. It focuses on both thermodynamic (e.g., density or enthalpy of vaporization) and dynamic properties, such as viscosity, self-diffusion coefficients, as well as diffusion coefficients of simple ions in both solvents. Emphasis is given to the effect of such properties on the suitability of the solvents for ion solvation and transfer processes, and the results are compared to experimental data whenever available. As well as providing reference data on the pure liquids, this study represents the first step in an ongoing comprehensive MD study of the interfaces between water and both solvents. 
TABLE 1: Intermolecular Potential Parameters for NPOE and OPLS NB

\begin{tabular}{lccc}
\hline atom & $\sigma(\mathrm{nm})$ & $\epsilon(\mathrm{kJ} / \mathrm{mol})$ & $q$ \\
\hline $\mathrm{O}_{\mathrm{N}}$ & 0.296 & 0.71128 & -0.370 \\
$\mathrm{~N}$ & 0.325 & 0.50208 & +0.650 \\
$\mathrm{C}_{\mathrm{N}}$ & 0.355 & 0.29288 & +0.090 \\
$\mathrm{C}_{\mathrm{A}}$ & 0.355 & 0.29288 & -0.115 \\
$\mathrm{H}_{\mathrm{A}}$ & 0.242 & 0.12552 & +0.115 \\
$\mathrm{C}_{\mathrm{O}}$ & 0.355 & 0.29288 & +0.085 \\
$\mathrm{O}_{\mathrm{E}}$ & 0.290 & 0.58576 & -0.285 \\
$\mathrm{C}_{\mathrm{E}}$ & 0.350 & 0.27614 & +0.140 \\
$\mathrm{H}_{\mathrm{E}}$ & 0.250 & 0.12552 & +0.030 \\
$\mathrm{C}$ & 0.350 & 0.27614 & -0.120 \\
$\mathrm{H}, \mathrm{H}_{\mathrm{T}}$ & 0.250 & 0.12552 & +0.060 \\
$\mathrm{C}_{\mathrm{T}}$ & 0.350 & 0.27614 & -0.180
\end{tabular}

\section{Potentials and Methods}

There have been two previous potential models proposed specifically for molecular simulations of NB. Both are all-atom potentials, where each atom is represented by a Lennard-Jones (L-J) center and a fixed-point charge. The first model is due to Michael and Benjamin (MB), ${ }^{14}$ who have taken charges obtained from ab initio calculations by Shlyapochnikov et al. ${ }^{19}$ and rescaled them to match the experimental dipole moment of the nitrobenzene molecule. The authors further tuned the charges and the L-J parameters to reproduce the experimental enthalpy of vaporization. The L-J cross-interaction parameters were calculated from the Lorentz-Berthelot combining rules. The model is fully flexible, with harmonic bond stretching and angle bending terms, as well as proper and improper torsional terms, all taken from the AMBER force field. ${ }^{20}$ It yields satisfactory agreement with experimental values for the dielectric constant and self-diffusion coefficient of the bulk liquid.

The second model is due to Janssen et al. (JAN) ${ }^{13}$ and is also based on the work by Shlyapochnikov et al. ${ }^{19}$ Its charges were calculated from a Mulliken population analysis, and also rescaled to match the experimental dipole moment. The L-J parameters were taken from literature values for benzene and the nitro group, and were not further optimized. Both bonds and angles were fixed, but a bistable torsional potential was implemented for the $\mathrm{NO}_{2}$ group.

Apart from these two potentials, one can also use the generalpurpose transferable force fields, such as AMBER, ${ }^{20}$ OPLS, ${ }^{21}$ CHARMM, ${ }^{22}$ etc., but only OPLS has included NB simulations in its parameter optimization procedure. For instance, Price et al. ${ }^{17}$ simulated NB using the OPLS-AA potential and obtained values for the density, enthalpy of vaporization and heat capacity in good agreement with experimental data. Price and Brooks ${ }^{18}$ have improved the agreement between simulated and experimental enthalpies of vaporization and free energies of hydration, by slightly increasing the L-J well depth of the nitrogen atom.

To further check the ability of these potential models, the simulations of the pure NB liquid were performed using all three potentials. As for the NPOE molecule, because there are no previously reported simulations, the OPLS-AA force field ${ }^{17,21}$ was applied for the bulk liquid simulations. The L-J parameters and point charges for NPOE are given in Table 1. In all cases, the bonds of the liquid molecules were considered fixed and the angles and dihedrals fully flexible. Further, improper dihedrals were added to keep the aromatic rings planar. Thus, the total potential is a sum of Lennard-Jones, electrostatic, angle bending and proper and improper torsional terms. A schematic diagram of the NB and NPOE molecules, showing all the labels used (for atoms and dihedrals), is given in Figure 1.

The MD simulations were carried out using version 3.3 of the GROMACS software package, ${ }^{23,24}$ during which all bond lengths were constrained using the LINCS algorithm. ${ }^{25}$ The equations of motion were integrated with the Verlet leapfrog algorithm, ${ }^{26}$ using a time step of 2 fs. The temperature $(T)$ was kept fixed at $298 \mathrm{~K}$ using the Nosé-Hoover (NH) thermostat, ${ }^{27,28}$ and whenever necessary, the pressure $(P)$ was held constant at 1 bar by the Parrinhelo-Raman (PR) coupling scheme. ${ }^{29}$ The NH scheme resorted to a coupling constant of $0.5 \mathrm{ps}$, whereas the PR used a coupling constant of $1.0 \mathrm{ps}$ (except for NPOE, where a constant of 3.0 ps was necessary) and an isothermal compressibility of $4.5 \times 10^{-5} \mathrm{bar}^{-1}$. Periodic boundary conditions were applied in all three Cartesian directions. The particle-mesh method of the Ewald sum ${ }^{30}$ was applied to deal with the long-range electrostatic forces, whereas the L-J interactions were handled using a twin range cutoff. Both the real-space part of the Ewald sum and the short-range L-J interactions, up to a distance of $1.1 \mathrm{~nm}$, were calculated with the help of a neighbor list, updated every 10 time steps. L-J interactions between 1.1 and $1.5 \mathrm{~nm}$ were also calculated every 10 steps and added to the total energy. In addition, a longrange dispersion correction was applied to both energy and pressure.

Thermodynamic and structural properties of both liquids were calculated from simulations of bulk systems in the NPT ensemble. The bulk systems contained either 246 NB molecules or 200 NPOE molecules placed in periodic cubic boxes. Following an energy minimization of at least 1000 steepest descent steps, the systems were equilibrated in the NPT ensemble until the observables fluctuated around their equilibrium values. This equilibration stage was much longer for NPOE (about $5 \mathrm{~ns}$ ) than for nitrobenzene (1 ns), due to the bulky nature of the former (see discussion below). Finally, production runs of $2 \mathrm{~ns}$ were performed for data collection. Properties were accumulated in blocks of 200 ps, from which total averages and standard deviations for each run were computed.

The diffusion coefficients of $\mathrm{Cl}^{-}$and $\mathrm{K}^{+}$ions in the pure liquids were evaluated from simulations in the NVT ensemble. Because we have observed that the mean square displacement of a single ion in a liquid showed a large amount of scatter, two long production runs of $10 \mathrm{~ns}$ were performed for each ion-solvent pair to improve the statistics. Furthermore, sampling was improved by including four ions in the simulation box, and by calculating the averages for all ions over both runs, using blocks of $200 \mathrm{ps}$. This is acceptable, provided the ions do not interact with each other. We have confirmed that this was the case by analyzing the ion-ion radial distribution functions (RDF) obtained from the MD simulations. In all cases, these RDFs show no interactions between ions at distances below 1 $\mathrm{nm}$. To set up the systems, four $\mathrm{Cl}^{-}$or four $\mathrm{K}^{+}$ions were randomly distributed inside liquid boxes that reproduce the equilibrium average density of the pure liquid, taken from the previous NPT runs. Following an energy minimization of at least 1000 steps, the systems were allowed to equilibrate for 500 ps, after which the production runs started. Using this procedure, we have obtained smooth plots of the mean square displacement, with significant linear portions. Diffusion coefficients were calculated from a linear fit to these portions, following the Einstein relation. The $\mathrm{K}^{+}$and $\mathrm{Cl}^{-}$ions were modeled by a L-J center and a unit point charge, according to the OPLS potential. ${ }^{31,32}$

For the shear viscosity calculations, we have employed the nonequilibrium periodic perturbation method. ${ }^{33}$ The method was described in detail by Hess, ${ }^{34}$ so we will limit ourselves to a brief outline. We perform a simulation in the NVT ensemble but add a periodic acceleration in the $x$ direction, which is a 

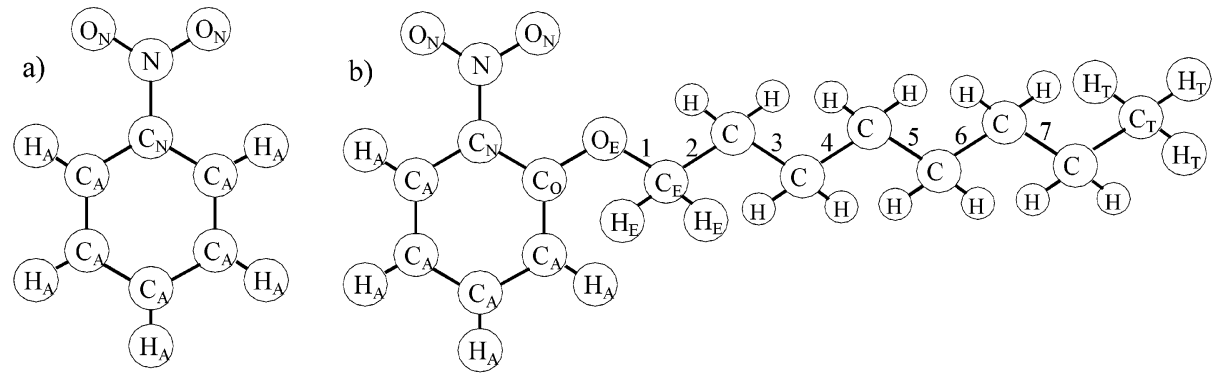

Figure 1. Schematic diagram of the (a) NB and (b) NPOE molecular models, showing the labels used for the atom types and dihedrals.

TABLE 2: Thermodynamic Properties for the NB and NPOE Liquids

\begin{tabular}{|c|c|c|c|c|c|c|}
\hline \multirow[b]{2}{*}{ property } & \multicolumn{4}{|c|}{ NB } & \multicolumn{2}{|c|}{ NPOE } \\
\hline & experiments & OPLS $^{21}$ & $\mathrm{MB}^{14}$ & $\mathrm{JAN}^{13}$ & experiments & OPLS \\
\hline$\rho\left(\mathrm{kg} \mathrm{m}^{-3}\right)$ & $1198.4^{a}$ & $1174.0 \pm 0.8$ & $1163.9 \pm 1.3$ & $1140.8 \pm 1.1$ & $1041^{a}$ & $1037.2 \pm 0.9$ \\
\hline$\Delta H_{\text {vap }}\left(\mathrm{kJ} \mathrm{mol}^{-1}\right)$ & $55.02^{b}$ & $54.25 \pm 0.40$ & $48.84 \pm 1.40$ & $35.72 \pm 0.42$ & $106.4^{c}$ & $105.32 \pm 0.85$ \\
\hline$\alpha\left(10^{-10} \mathrm{~Pa}^{-1}\right)$ & $5.23^{c}$ & $5.09 \pm 0.56$ & $6.19 \pm 0.88$ & $5.78 \pm 0.65$ & & $4.37 \pm 0.38$ \\
\hline$\mu$ (Debye) & $4.22^{c}$ & 3.30 & 4.35 & 4.44 & & 4.33 \\
\hline
\end{tabular}

${ }^{a}$ Reference 5. ${ }^{b}$ Reference 36. ${ }^{c}$ Reference 3.

function of the $z$ coordinate only. This acceleration term has the following form:

$$
a_{x}(z)=A \cos \left(\frac{2 \pi}{L_{z}} z\right)
$$

where $L_{z}$ is the length of the box in the $z$ direction, and the amplitude $A$ is a tunable parameter. The generated velocity profile $(v)$ is computed during the simulation, and the shear viscosity $(\eta)$ is calculated from

$$
\eta=\frac{A \rho}{v}\left(\frac{L_{z}}{2 \pi}\right)^{2}
$$

where $\rho$ is the system density. The value of $A$ should be a compromise: if it is too low, the statistics on $v$ are poor; if it is too high, the shear rate is too high and the system moves too far from equilibrium. ${ }^{34}$ In practice, because $v$ will depend on the square of $L_{z}$, the ideal box is elongated in the $z$ direction. This was accomplished here by taking a given configuration for each pure liquid from the previous NPT runs, and multiplying it three times in the $z$ direction. A small gap of $1 \mathrm{~nm}$ was allowed between replicas to avoid molecule overlap. After a quick energy minimization, the elongated boxes were equilibrated in the NPT ensemble for 200 ps. Finally, a configuration with the same density as the average value of each pure liquid was chosen and used as the starting point for a 2 ns run in the NVT ensemble with the added acceleration term. The first 200 ps of this run were discarded and the remainder divided into

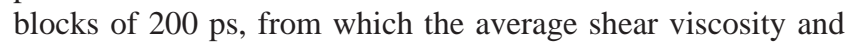
standard deviation were computed.

\section{Results and Discussion}

In the following, we first focus on the thermodynamic and structural properties of both the NB and NPOE pure liquids, at $298 \mathrm{~K}$ and 1 bar, and then move on to dynamic properties, ending with a discussion of the diffusion of simple ions in the bulk liquids.

3.1. Thermodynamics and Structure. The calculated values for the density, enthalpy of vaporization $\left(\Delta H_{\mathrm{vap}}\right)$, isothermal compressibility $(\alpha)$ and dipole moment $(\mu)$ of NB and NPOE are presented in Table 2, along with the experimental results. The enthalpy of vaporization was calculated according to the Jorgensen approach, ${ }^{35}$ which assumes that the sum of the vibrational terms with the kinetic energy is the same in the liquid and gas phases. With this assumption, $\Delta H_{\text {vap }}$ can be estimated from

$$
\begin{aligned}
\Delta H_{\text {vap }}=E_{\text {dih }}(\mathrm{g})+ & E_{\text {intra }}(\mathrm{g})- \\
& {\left[E_{\text {dih }}(1)+E_{\text {intra }}(1)+E_{\text {inter }}(1)\right]+R T }
\end{aligned}
$$

where $E_{\text {dih }}$ is the dihedral energy (including $1-4$ terms), $E_{\text {intra }}$ is the interaction energy between atoms on the same molecule separated by more that three bonds, and $E_{\text {inter }}$ is the intermolecular energy.

All the applied OPLS, MB and JAN potential models underestimate the density of the NB liquid, but the OPLS potential affords the best agreement (error of $2.5 \%$ ). The same is true for the enthalpy of vaporization, giving OPLS in this case an error of only $1.4 \%$. On the other hand, the isothermal compressibility is well reproduced by all models (though slightly higher for MB) and, once again, the best result is obtained with OPLS. The only exception to this rule is the molecular dipole moment, for which the OPLS model predicts a much lower value (by more than 20\%) than experiment. One must bear in mind that the experimental dipole moment values reported in Table 2 were obtained in the gas phase-in the liquid phase, these are likely to be slightly higher. In practice this means that it is very likely that all models are underestimating this property and that this underestimation in the case of OPLS might be even higher than apparent. In fact, the latter model is based on transferable parameters, which are optimized for a wide range of molecular species, but not specifically for nitrobenzene. Contrastingly, the charges of both the MB and the JAN models were rescaled to match the experimental dipole moment of nitrobenzene and therefore afford a much better agreement regarding this property. It is also worth mentioning that we have examined the effect of the system size on the calculated properties, by running simulations with different numbers $(N=27,46,90$, and 155) of NB molecules. The values of thermodynamic properties obtained from the simulations with 90 molecules or more show errors below $0.3 \%$, relative to those of the largest system $(N=$ 246). This means that we can be confident that the results presented in Table 2 are free from finite-size effects.

With respect to NPOE, our simulations show excellent agreement with the experimental values for the density and $\Delta H_{\text {vap. }}$ This is remarkable considering that there have been no 


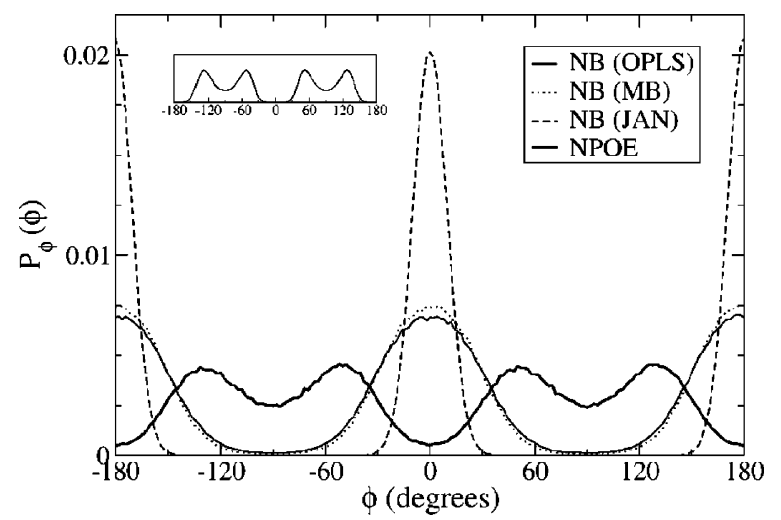

Figure 2. Probability distributions for the nitro group dihedral of the NB and NPOE molecules. Inset: Boltzmann factor calculated for NPOE from the sum of the dihedral potential with the L-J potential for the third and fourth neighbors of the $\mathrm{O}_{\mathrm{N}}$ atoms.

previous simulations of this liquid. Unfortunately, no experimental data were found for the compressibility or the dipole moment of NPOE. However, it is worth noting that the latter is very close to the value for nitrobenzene, a fact that may well explain the reported similarities in the solvation properties of both liquids. ${ }^{11,12}$ Finite-size effects were also investigated by running simulations with $20,38,66$, and 106 NPOE molecules. Once again, the results show that the larger $N=200$ system is free of finite-size effects.

The structural properties of the NB and NPOE liquids were characterized in terms of angle and dihedral probability distribution functions, RDFs and dipole orientation correlation functions. Notice that we have examined many such distributions, but only the most interesting ones, i.e., those that better describe the most important aspects of the structure of NB and NPOE, will be presented here.

Let us first compare the three models of NB with each other and with the aromatic part of NPOE. From the angle and dihedral distributions, we could see that the aromatic ring of both nitrobenzene and NPOE is essentially flat, with most angles and dihedrals showing very similar distributions in all models. A notable exception is the $\mathrm{C}_{\mathrm{A}}-\mathrm{C}_{\mathrm{N}}-\mathrm{N}-\mathrm{O}_{\mathrm{N}}$ dihedral (see Figure 1 ), the distribution of which is plotted in Figure 2. All models of $\mathrm{NB}$ lead to distributions with peaks at 0 and $180^{\circ}$, corresponding to an alignment of the nitro group parallel to the aromatic ring plane. However, the peaks are much sharper for the JAN model than for the other two models, which is evidence of a reduced rotational mobility of the nitro group in the former case. This is a direct consequence of the increased torsional barrier implemented in the JAN potential, to favor planar conformations. ${ }^{13}$ The most interesting aspect in Figure 2, however, is the difference between the distributions for NPOE and NB. In most NPOE configurations, the nitro group is tilted relative to the benzene plane, with maxima at about \pm 50 and $\pm 130^{\circ}$, instead of lying at the minima of the dihedral potential. Furthermore, the same dihedral distribution computed in the gas phase (data not shown) is very similar to the liquid-phase distribution of Figure 2, which rules out any important effect of intermolecular interactions. Considering this, the most probable cause for the observed tilting of the dihedral is the strong repulsion between the oxygens of the nitro group and the oxygen atom of the ether chain (see Figure 1b)-the nitro group assumes the position that minimizes the sum of torsional and L-J potentials. In fact, a rough calculation of the Boltzmann factor as a function of dihedral angle, taking into account only the dihedral potential and the L-J interactions of the nitrooxygens with their third and fourth nearest neighbors, closely
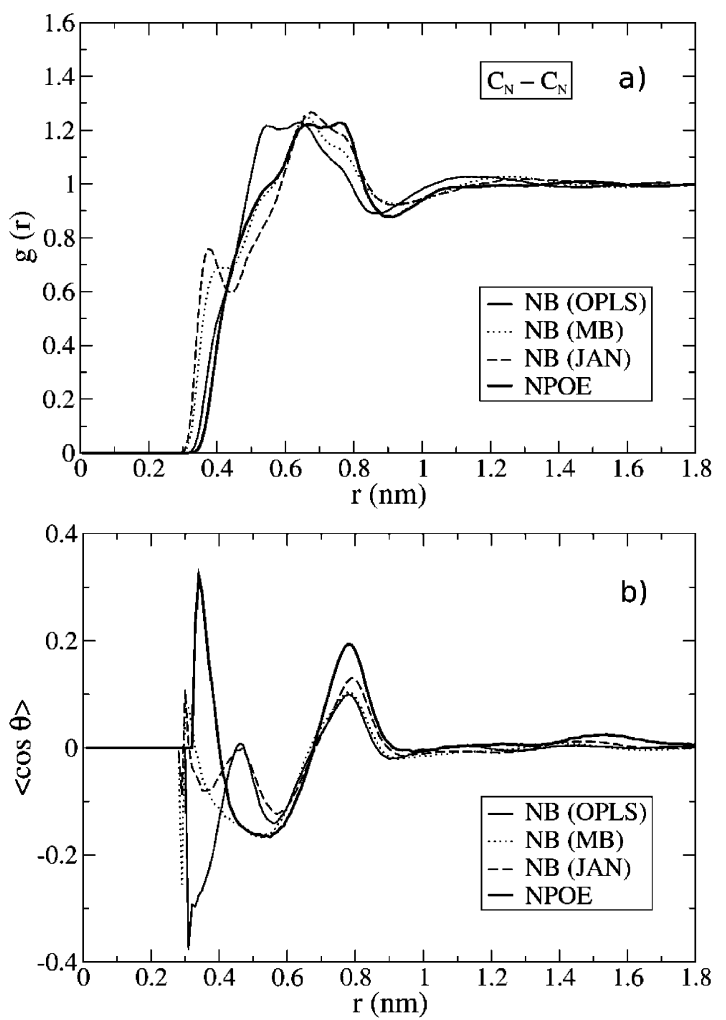

Figure 3. $\mathrm{C}_{\mathrm{N}}-\mathrm{C}_{\mathrm{N}}$ radial distribution functions (a) and dipole orientation correlation functions (b) for the NB and NPOE liquids.

reproduces the shape of the NPOE dihedral distribution (see inset in Figure 2).

Because the center of mass of the nitrobenzene molecule practically coincides with the carbon atom bonded to the nitro group $\left(\mathrm{C}_{\mathrm{N}}\right)$, this atom was used as a basis to analyze the structure of the NB liquid. The $\mathrm{C}_{\mathrm{N}}-\mathrm{C}_{\mathrm{N}}$ RDFs for NB (Figure 3a) show a complex structure, where one can distinguish three overlapping peaks, at approximately $0.55,0.66$, and $0.76 \mathrm{~nm}$. These vary in strength according to the model used: the first peak is higher with the OPLS potential, followed by the MB and the JAN potentials, whereas the opposite trend is observed in the other two peaks. Most likely, there is a link between the relative importance of these peaks and the equilibrium liquid density for the three potential models. The model that predicts a higher density (closer to the experimental value), OPLS, also shows closer packing of the nitrobenzene molecules as revealed by its higher RDF first peak. The JAN model shows the loosest packing arrangement (higher third peak and lowest first peak), corresponding to the lowest equilibrium density. Notice also that the liquid retains some spatial correlations up to separations of about $1.4 \mathrm{~nm}$, which justifies the cutoff of $1.5 \mathrm{~nm}$ employed in our simulations.

Further insight into the NB liquid structure can be obtained by comparing the $\mathrm{C}_{\mathrm{N}}-\mathrm{C}_{\mathrm{N}} \mathrm{RDF}$ with the dipole orientation correlation function based on the relative distances between the $\mathrm{C}_{\mathrm{N}}$ atoms (Figure $3 \mathrm{~b}$ ). Despite the difficulties associated with drawing conclusions about the preferred orientation of the molecules from average values of the dipole vector orientations, one can nevertheless distinguish some clear trends. Thus, NB molecules in the first peak show a tendency for antiparallel dipolar alignment, whereas those in the third peak have a tendency for parallel dipolar alignment. As for the second peak, the value close to zero probably reflects a preference for perpendicular alignment. Another feature that might be observed in the JAN model RDF is a peak at very short molecular 

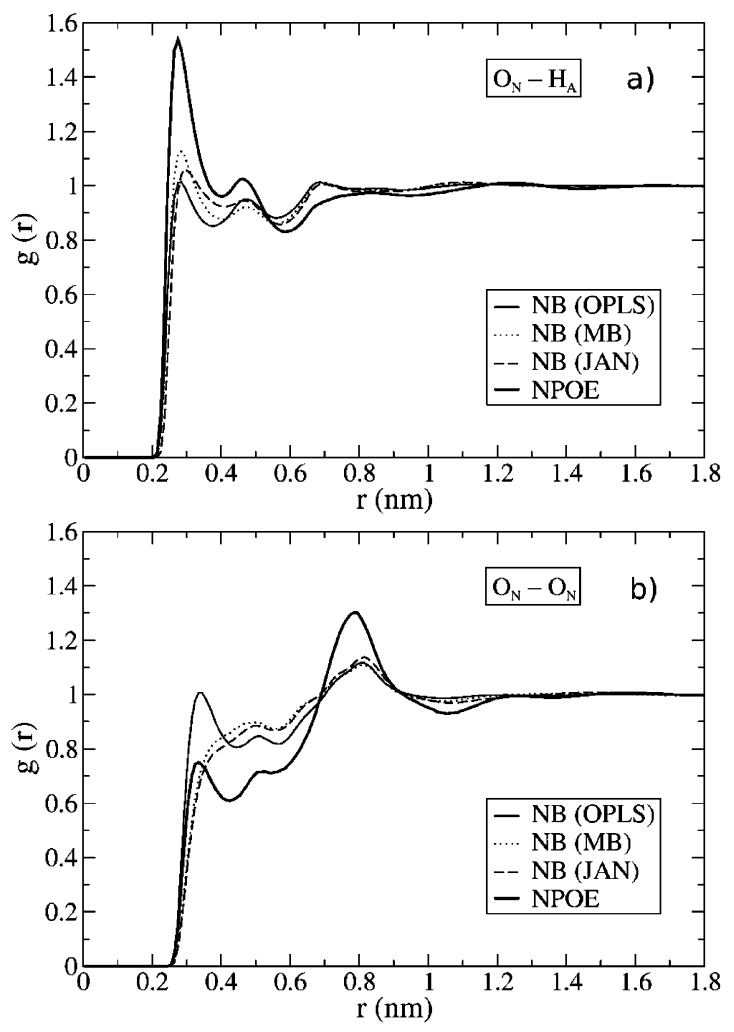

Figure 4. $\mathrm{O}_{\mathrm{N}}-\mathrm{H}_{\mathrm{A}}$ (a) and $\mathrm{O}_{\mathrm{N}}-\mathrm{O}_{\mathrm{N}}$ (b) radial distribution functions for the NB and NPOE liquids.

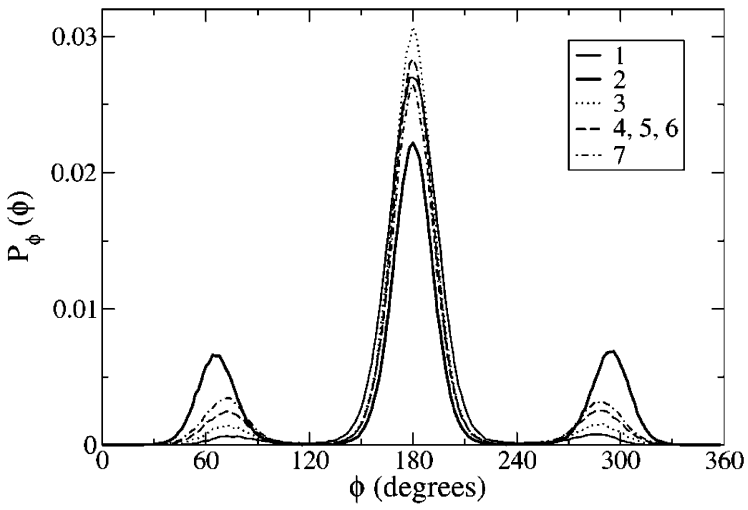

Figure 5. Dihedral probability distributions for the NPOE chain. The lines correspond to the dihedrals as numbered in Figure 1.

separations (about $0.38 \mathrm{~nm}$ ), which becomes a shoulder in the MB model and is almost indistinguishable in the OPLS model. This feature has been assigned by Janssen et al. ${ }^{13}$ to pairs of nitrobenzene molecules with antiparallel dipolar alignment, and such preferred alignment can also be noticed in Figure $3 \mathrm{~b}$.

As for NPOE, the $\mathrm{C}_{\mathrm{N}}-\mathrm{C}_{\mathrm{N}} \mathrm{RDF}$ is very similar to that of nitrobenzene, but with a predominance of the third peak over the first (i.e., looser packing of the aromatic rings). Furthermore, there is no evidence of dimer formation, because there is no shoulder in the RDF at short separation distances. Contrarily to nitrobenzene, two aromatic rings of NPOE at very short distances show a tendency for parallel dipolar alignment (see Figure 3b). This behavior can be attributed to steric effects arising from the aliphatic NPOE chains, which render direct interactions between aromatic rings more difficult. The average number of neighbors for the first coordination shell may be estimated by integrating the $\mathrm{C}_{\mathrm{N}}-\mathrm{C}_{\mathrm{N}} \mathrm{RDF}$ up to its first minimum $(\sim 0.9 \mathrm{~nm})$. For NPOE, the calculated value is 7.5 , to
TABLE 3: Dihedral Probabilities for the NPOE Chain

\begin{tabular}{cc}
\hline dihedral $^{a}$ & trans probability \\
\hline 1 & 0.950 \\
2 & 0.628 \\
3 & 0.905 \\
4 & 0.841 \\
5 & 0.853 \\
6 & 0.845 \\
7 & 0.792
\end{tabular}

${ }^{a}$ Dihedrals numbered as in Figure 1.

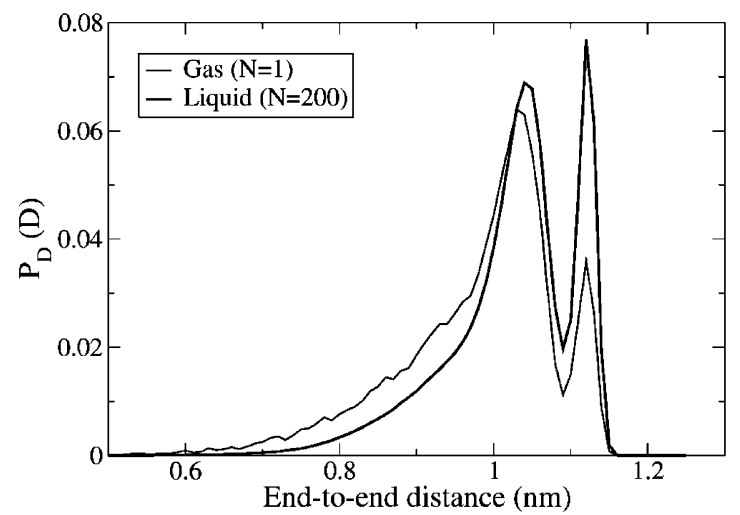

Figure 6. Probability distributions for the NPOE chain end-to-end distance in the gas and liquid phases.

be compared with the value of 16.5 obtained for NB by all model potentials.

Let us now look to the most important RDFs pertaining to the nitro group. The $\mathrm{O}_{\mathrm{N}}-\mathrm{H}_{\mathrm{A}} \mathrm{RDF}$ (Figure 4a) exhibits a strong peak at $0.275 \mathrm{~nm}$ and a secondary peak at $0.47 \mathrm{~nm}$, indicating strong interactions between these atoms. Actually, the interactions between the nitro group and the aromatic hydrogens dominate all the RDFs involving atoms in these groups (data not shown). Integration of the first peak of this RDF (up to 0.4 $\mathrm{nm}$ ) yields an estimate of the average number of $\mathrm{H}_{\mathrm{A}}$ atoms around each $\mathrm{O}_{\mathrm{N}}$. This number is approximately 5 for all models of NB, but only 2 for NPOE, possibly due to steric hindrances caused by the presence of the aliphatic chains. Further, the computed $\mathrm{O}_{\mathrm{N}}-\mathrm{O}_{\mathrm{N}} \mathrm{RDFs}$ (Figure $4 \mathrm{~b}$ ) enabled us to evaluate the associations between the most polar groups of two adjacent molecules. The RDFs for NB are all very similar, except for a small peak at $0.34 \mathrm{~nm}$ present in the OPLS model but absent in the other two. This means that the OPLS model allows for some mutual interaction between the nitro groups of two adjacent molecules, which might be due to its lower dipole moment (see Table 2). NPOE also shows such a peak, although it is much weaker.

Moving on to the aliphatic part of NPOE, it is interesting to assess the extent of molecular folding of the chain. This can be accomplished by examining the dihedral distributions for all rotating bonds in the chain (Figure 5). The percentages of gauche and trans conformations for each dihedral, obtained from integration of the peaks in Figure 5, are shown in Table 3. One can see that the $\mathrm{C}_{\mathrm{O}}-\mathrm{O}_{\mathrm{E}}-\mathrm{C}_{\mathrm{E}}-\mathrm{C}$ dihedral (dihedral 1 in Figure 1b) is almost entirely in the trans configuration (over 95\%), whereas the $\mathrm{O}_{\mathrm{E}}-\mathrm{C}_{\mathrm{E}}-\mathrm{C}-\mathrm{C}$ dihedral (dihedral 2) shows the highest percentage of gauche conformations $(37 \%)$. The other dihedrals show very similar intermediate behavior. Overall, the chain shows a small extent of folding, with approximately $26 \%$ of all chains fully stretched. We have also calculated the probability distribution for the distance between the $\mathrm{C}_{\mathrm{O}}$ and $\mathrm{C}_{\mathrm{T}}$ atoms of NPOE (see Figure 1b). The distributions for the liquid and gas phases are plotted in Figure 6; they show two distinct 
TABLE 4: Dynamic Properties for the NB and NPOE Liquids

\begin{tabular}{|c|c|c|c|c|c|c|}
\hline \multirow[b]{2}{*}{ property } & \multicolumn{4}{|c|}{ NB } & \multicolumn{2}{|c|}{ NPOE } \\
\hline & experiments & OPLS $^{21}$ & $\mathrm{MB}^{14}$ & $\mathrm{JAN}^{13}$ & experiments & OPLS \\
\hline$\eta\left(10^{-3} \mathrm{~kg} \mathrm{~m}^{-1} \mathrm{~s}^{-1}\right)$ & $1.795^{a}$ & $1.744 \pm 0.11$ & $1.449 \pm 0.08$ & $1.199 \pm 0.06$ & $13.80^{a}$ & $16.94 \pm 2.74$ \\
\hline$D^{\infty}\left(10^{-9} \mathrm{~m}^{2} \mathrm{~s}^{-1}\right)$ & $1.080^{b}$ & $0.945 \pm 0.045$ & $1.030 \pm 0.087$ & & & $0.068 \pm 0.020$ \\
\hline$D_{\mathrm{Cl}}^{\infty}\left(10^{-9} \mathrm{~m}^{2} \mathrm{~s}^{-1}\right)$ & $0.460^{c}$ & $0.487 \pm 0.048$ & $0.575 \pm 0.065$ & & $0.060^{c}$ & $0.028 \pm 0.009$ \\
\hline$D_{\mathrm{K}}^{\infty}\left(10^{-9} \mathrm{~m}^{2} \mathrm{~s}^{-1}\right)$ & $0.475^{c}$ & $0.364 \pm 0.035$ & $0.486 \pm 0.077$ & & $0.062^{c}$ & $0.028 \pm 0.010$ \\
\hline
\end{tabular}

${ }^{a}$ Reference 5. ${ }^{b}$ Reference $40 .{ }^{c}$ Reference 6.

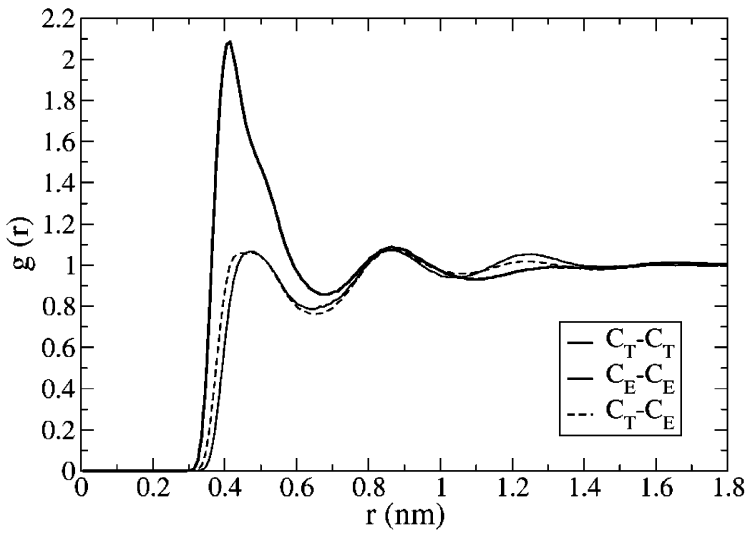

Figure 7. Radial distribution functions between carbons on the two ends of the NPOE chain.

peaks, one at $1.04 \mathrm{~nm}$ and another at 1.12. The latter value matches that of a fully stretched chain $(1.122 \mathrm{~nm})$. In fact, integration of the last peak for the liquid phase yields a probability of 0.24 , which agrees with the percentage of fully stretched chains computed from the dihedral distributions. The location of the first peak matches closely the end-to-end distance of a chain with dihedral 2 in a gauche position $(1.042 \mathrm{~nm})$. This also makes sense because this is the dihedral with the highest gauche probability (Table 3 ). Chains in a terminalgauche conformation (end-to-end distance of $1.052 \mathrm{~nm}$ ) are also frequent and form the shoulder in the distribution at approximately $1.05 \mathrm{~nm}$. The first peak decays rapidly at short distances, confirming the low extent of chain folding for NPOE. The distribution for the gas phase is qualitatively similar but shows a lower percentage of fully stretched chains. This is presumably due to the absence of packing constraints in the gas phase. These results are in qualitative agreement with other simulation studies of the liquids decane ${ }^{37,38}$ and 1-octanol, ${ }^{39}$ thus suggesting that the NPOE chain behaves much like a linear alkane chain.

Finally, Figure 7 shows the RDFs involving the first $\left(\mathrm{C}_{\mathrm{E}}\right)$ and last $\left(\mathrm{C}_{\mathrm{T}}\right)$ aliphatic carbon atoms in the NPOE chain. All RDFs have similar shapes, with primary and secondary peaks. However, the first peak of the $\mathrm{C}_{\mathrm{T}}-\mathrm{C}_{\mathrm{T}} \mathrm{RDF}$ is much stronger and appears at closer distances (at $0.41 \mathrm{~nm}$ compared to 0.475 $\mathrm{nm}$ for the other RDFs). This shows the importance of steric effects on the solvation properties of NPOE. The terminal carbons are much more accessible and thus show stronger mutual interactions. On the other hand, access to the carbons closer to the ether oxygen is made difficult by the presence of the bulky polar aromatic ring. Another aspect worth noticing is the persistence of spatial correlations up to separations of about $1.5 \mathrm{~nm}$.

To summarize this section, the three different potential models for NB show some structural differences, namely with respect to the packing of the aromatic rings and to the interactions between the nitro groups. However, these differences are only present at short separation distances and have only a small impact on the calculated thermodynamic properties. Comparing

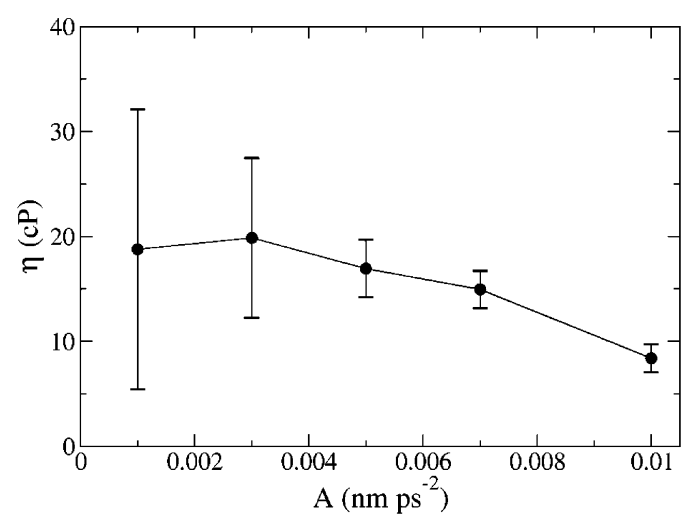

Figure 8. Calculated shear viscosity of NPOE as a function of acceleration amplitude.

these properties to experimental values, the OPLS model produces the most accurate results, provided one can live with the discrepancy in the dipole moment. The MB model is free of this problem and also yields a satisfactory comparison with experimental properties. As for NPOE, the structure of the aromatic ring is similar to that of $\mathrm{NB}$, except for the tilting of the nitro group relative to the aromatic plane. The structure of the liquid is dominated, on one hand, by the interactions between the positive and negative ends of the molecular dipole (located on the aromatic side) and, on the other hand, by the interactions between the aliphatic chains. These are rendered more difficult by the presence of the aromatic ring, but that seems to have little effect on the degree of folding (relative to linear alkanes).

3.2. Dynamic Properties. The results obtained for the viscosity of NB and NPOE, using the nonequilibrium MD method described in section 2, are shown in Table 4. As mentioned previously, the value of the acceleration amplitude, $A$, must be chosen with care. In the case of NB, the calculated viscosity was found to be the same, within statistical error, for amplitudes of $0.01,0.007$, and $0.005 \mathrm{~nm} / \mathrm{ps}^{2}$. The values reported in Table 4 are for $A=0.01 \mathrm{~nm} / \mathrm{ps}^{2}$, because the statistical errors are the smallest for this amplitude. In the case of NPOE, the calculated viscosities exhibit a strong dependence on $A$ for the values examined, as shown in Figure 8. For amplitudes of 0.005 $\mathrm{nm} / \mathrm{ps}^{2}$ and below, the results agree within statistical error. However, this error is proportional to the inverse of the amplitude, and it is already very large for the two smallest amplitudes. For that reason, the value calculated for $A=0.005$ $\mathrm{nm} / \mathrm{ps}^{2}$ should be considered as the most reliable. One might reduce the error, thus allowing for lower amplitudes, by expanding the box in the $z$ direction even further. However, that would considerably increase the computation time of these already expensive simulations.

As can be seen, the OPLS model provides remarkable agreement with the experimental viscosity, followed by the MB and the JAN models, in that order. The value computed for the viscosity of NPOE is almost 10 times higher than that of nitrobenzene, and fairly close to the experimental result. This much higher viscosity is very likely related to the structure of 


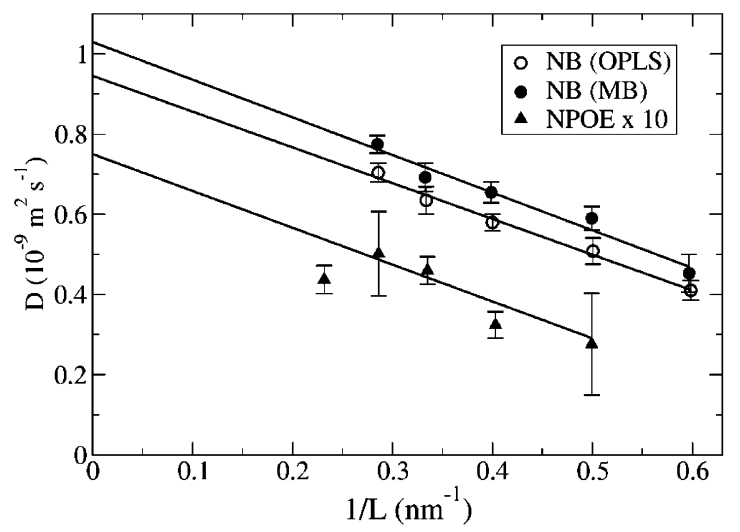

Figure 9. Self-diffusion coefficients as a function of inverse box length The NPOE results are multiplied by 10 for an easy visualization. The lines are linear fits to the data, extrapolated to infinite box size.

the NPOE molecule. On one hand, it is not as compact as the nitrobenzene molecule, which is shaped like an ellipsoid. On the other hand, the presence of the bulky aromatic group prevents the aliphatic chains from sliding past each other like in the case of linear alkanes. The combination of an almost spherical aromatic ring with a long aliphatic chain causes molecular entanglement and produces a sluggish response to shear. As will be shown below, this high viscosity has a marked effect on the diffusive motion inside the liquid.

Unlike the viscosity, the self-diffusion coefficient $(D)$ of liquids suffers from significant finite-size effects, normally decreasing with the inverse of the linear box dimension. ${ }^{40,41}$ This was checked here by computing the self-diffusion coefficients of the two liquids, using systems with different numbers of molecules (i.e., those referred to in section 3.1). For each case, the diffusion coefficient was obtained from a linear fit to the mean square displacement, averaged over all molecules. The results for nitrobenzene (OPLS and MB models) and NPOE are plotted in Figure 9 as a function of $L^{-1}$. Notice that the JAN model was excluded from this study (as well as from the following ones), because it showed the worst performance of all NB potentials in terms of thermodynamic properties and viscosity. One can confirm that there is indeed a linear relationship between $D$ and the inverse box dimension. Both the nitrobenzene models obey this relation very closely, whereas for NPOE there is a lot more scatter in the results. This is partly due to the much slower diffusion of this molecular liquid, which in turn makes the calculations less accurate. By fitting a straight line through the points, one can extrapolate to infinite system size, at $1 / L=0$. The values of the self-diffusion coefficient at infinite size $\left(D^{\infty}\right)$ obtained for each case are shown in Table 4. The results from both nitrobenzene models agree nicely with the experimental value, with a slightly better performance for the MB model. Even though there are no experimental data to compare with, the computed value for the diffusion coefficient of NPOE is reasonable, because it is about an order of magnitude below that of nitrobenzene. This correlates well with the differences in viscosity of the two liquids, described previously.

3.3. Solvation and Diffusion of Ions. We conclude this section by examining the diffusion of $\mathrm{Cl}^{-}\left(D_{\mathrm{Cl}}\right)$ and $\mathrm{K}^{+}\left(D_{\mathrm{K}}\right)$ ions in nitrobenzene, as well as in NPOE. Because finite-size effects should again be taken into account, but to avoid performing the same analysis as for the self-diffusion coefficients, which would be far too computationally demanding, we proceed as follows: each ion diffusion coefficient, calculated in a finite box filled with a given liquid, was divided by $D$ for that liquid with the same box size and multiplied by $D^{\infty}$ for the

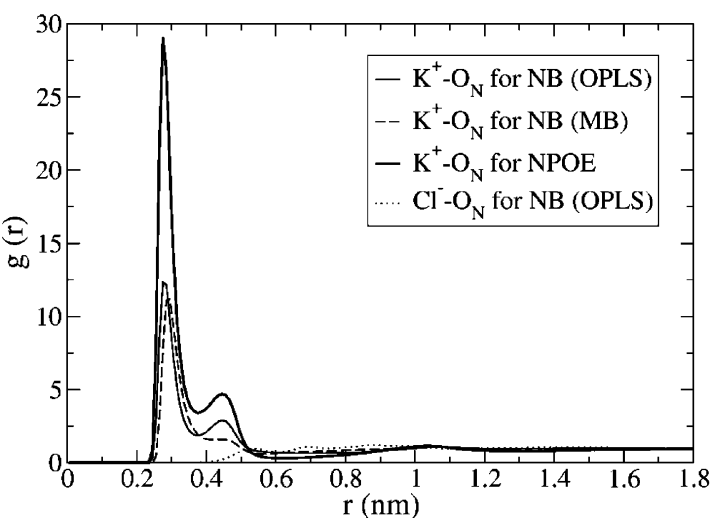

Figure 10. Ion $-\mathrm{O}_{\mathrm{N}}$ radial distribution functions for the $\mathrm{NB}$ and $\mathrm{NPOE}$ liquids.

same liquid. This procedure assumes that the finite-size correction to the diffusion coefficient depends only on the nature of the solvent. The corrected values for the ion diffusion coefficients are given in Table 4 . The first conclusion to be drawn is that the ions diffuse much faster (by more than an order of magnitude) in nitrobenzene than in NPOE. Once more, this is likely related to the increase of 1 order of magnitude in the viscosity of NPOE, relative to that of NB. For similar reasons, the ions diffuse slightly faster with the MB model than with OPLS, which is somewhat more dense and viscous. Nevertheless, both models yield results in good agreement with the experimental values. One can observe that the NPOE simulation values are slightly below the experimental ones. This is most probably due to approximations used in the experiments, namely applying the Stokes-Einstein relation for calculating the diffusion coefficients, on one hand, ${ }^{6}$ and underestimating the fact that the hydrophilic ions drag at least one solvation shell when going into the organic solvent, on the other hand. In terms of differences between the two ions, there is a slight tendency for $\mathrm{Cl}^{-}$to diffuse faster than $\mathrm{K}^{+}$. This trend goes against experimental observations, even though the differences between the ions are very small (and likely within experimental error). The difference between the diffusion coefficients of both ions is small in nitrobenzene but virtually disappears when the ions are dissolved in NPOE. In the latter case, the dynamics of the ions are much more conditioned by the high solvent viscosity, and any intrinsic difference between them becomes secondary.

More insight about the ion solvation process can be gained by considering the RDFs between the ions and the solvents. Figure 10 depicts the most pertinent $\mathrm{K}^{+}-\mathrm{O}_{\mathrm{N}}$ and $\mathrm{Cl}^{-}-\mathrm{O}_{\mathrm{N}} \mathrm{RDFs}$ for both NB and NPOE liquids. There is a strong interaction between the positively charged potassium ion and the electronegative oxygen atoms, manifested in a large peak at $0.28 \mathrm{~nm}$, whereas this interaction is absent in the case of chloride. From analysis of this and other RDFs, one can trace the general structure of the first solvation shell of $\mathrm{K}^{+}$. Each liquid molecule contributes with one $\mathrm{O}_{\mathrm{N}}$ atom located very close to the ion (first peak in Figure 10). The $\mathrm{O}_{\mathrm{N}}-\mathrm{N}$ bond is oriented perpendicularly to the ion surface, producing a single peak at $0.4 \mathrm{~nm}$ in the $\mathrm{K}^{+}-\mathrm{N}$ RDF (not shown) and the secondary peak in Figure 10 (at $0.45 \mathrm{~nm}$ ), for the other $\mathrm{O}_{\mathrm{N}}$ atom in the same molecule, as well as a single peak at $0.5 \mathrm{~nm}$ in the $\mathrm{K}^{+}-\mathrm{C}_{\mathrm{N}} \mathrm{RDF}$ (also not shown). This structure prevails in the two liquids and is more easily perceived by examining a snapshot of the molecules during the simulation (Figure 11a). Both snapshots shown in Figure 11 were obtained during MD runs of ion solvation by $\mathrm{NB}$, selecting only the solvent molecules that are within the 

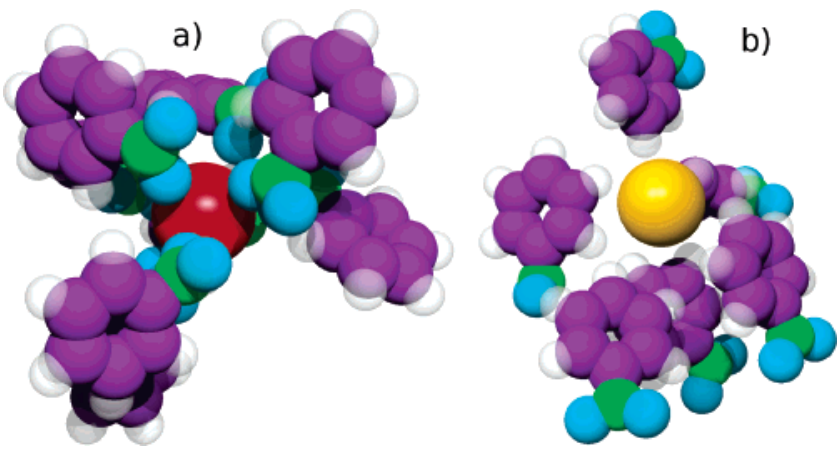

Figure 11. Snapshots of the potassium (a) and chloride (b) ion solvation shells in liquid nitrobenzene. The $\mathrm{K}^{+}$ion is represented by the solid red sphere, whereas the $\mathrm{Cl}^{-}$ion is the solid yellow sphere. The remaining spheres are nitrobenzene atoms: C, purple; N, green; $\mathrm{O}$, blue; $\mathrm{H}$, white (transparent).

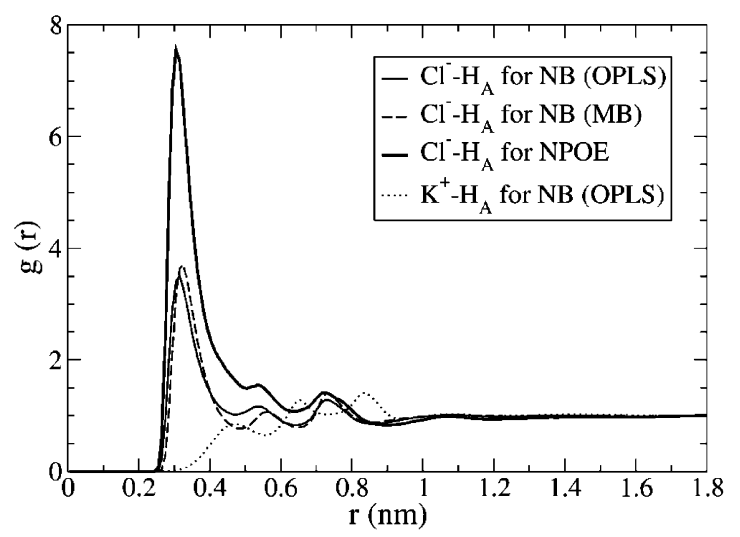

Figure 12. Ion $-\mathrm{H}_{\mathrm{A}}$ radial distribution functions for the NB and NPOE liquids.

ion solvation shell (some molecules in out-of-plane locations were removed so as not to obstruct the view of the ion). Figure 11a clearly shows the oxygen atoms of the nitro group pointing toward the ion, in agreement with the structure deduced from the RDFs. Integration of the first peak of the $\mathrm{K}^{+}-\mathrm{O}_{\mathrm{N}} \mathrm{RDF}$ yields the number of liquid molecules in the first shell of $\mathrm{K}^{+}$. This number is 8.9 for all models of NB and 8.2 for NPOE (corroborated by integration of the other mentioned RDFs).

In Figure 12, the $\mathrm{Cl}^{-}-\mathrm{H}_{\mathrm{A}}$ RDFs for nitrobenzene and NPOE are plotted, together with the $\mathrm{K}^{+}-\mathrm{H}_{\mathrm{A}} \mathrm{RDF}$ for nitrobenzene. In the $\mathrm{Cl}^{-}-\mathrm{H}_{\mathrm{A}} \mathrm{RDF}$, there is a large peak at $0.3 \mathrm{~nm}$, denoting a very strong interaction between this negatively charged ion and the positive partial charge on the hydrogens. As expected, the $\mathrm{K}^{+}-\mathrm{H}_{\mathrm{A}}$ show no such peak, due to the repulsive nature of the interactions between these atoms. The structure of the solvation shell of the chloride ions is somewhat more complicated than that of potassium, due to the larger variety of available conformations for the $\mathrm{Cl}^{-}-\mathrm{H}_{\mathrm{A}}$ interactions. From analysis of the several RDFs, one can infer that each liquid molecule contributes with two $\mathrm{H}_{\mathrm{A}}$ atoms in close vicinity to the ion. For NB, these are one hydrogen located at the meta position and one located at either the para or the ortho positions (with a slight preference for the former, because it is further away from the negative end of the dipole). This shell structure explains the three peaks in the $\mathrm{Cl}^{-}-\mathrm{H}_{\mathrm{A}} \mathrm{RDF}$, as well as those in the $\mathrm{Cl}^{-}-\mathrm{C}_{\mathrm{A}} \mathrm{RDF}$ (not shown), and is illustrated in Figure 11b. In this snapshot, one can clearly observe NB molecules with their meta and para hydrogens oriented toward the chloride ion (e.g., the three molecules located below the ion), as well as with their meta and ortho hydrogens (e.g., the molecule on the left of the ion). Integration of the first peak of the $\mathrm{Cl}^{-}-\mathrm{H}_{\mathrm{A}}$

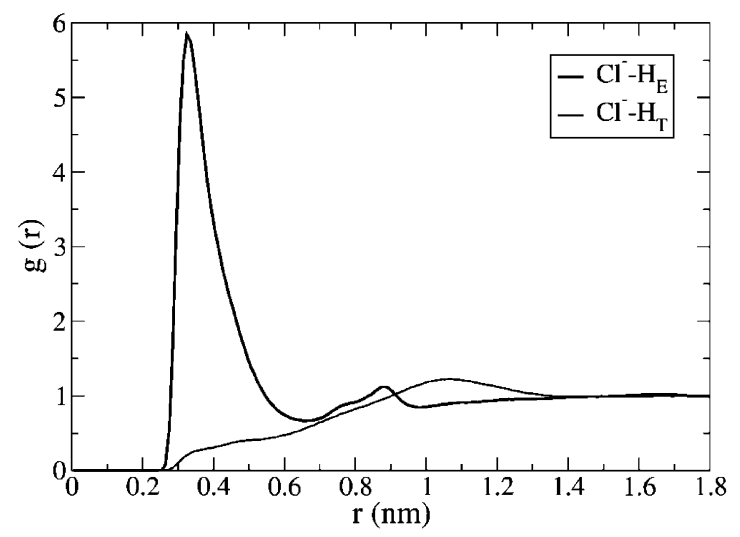

Figure 13. Radial distribution functions between $\mathrm{Cl}^{-}$ions and hydrogens on both ends of the NPOE chain.

RDF yields 7.7 NB molecules in the first solvation shell of the chloride ion, but only 5.1 NPOE molecules. This difference is explained by considering the interactions of this ion with the hydrogens from the aliphatic chain (Figure 13). The RDF between $\mathrm{Cl}^{-}$and the first hydrogens of the chain shows a strong peak at short distances, similar to that observed with the aromatic hydrogens. However, this peak is absent in the RDF relative to the last hydrogens of the chain. Integration of the first RDF peak yields 2.5 NPOE molecules, thus yielding a total of 7.6 NPOE molecules solvating the $\mathrm{Cl}^{-}$ion, similarly to NB. It is worth mentioning that the experimental values of standard Gibbs energies of transfer of $\mathrm{K}^{+}$and $\mathrm{Cl}^{-}$from water to $\mathrm{NB}$ are slightly smaller than the corresponding ones from water to NPOE. 1,2,6 Besides, the experimental standard Gibbs energies of transfer of $\mathrm{K}^{+}$from water to both NB and NPOE are lower than that of $\mathrm{Cl}^{-}$ion from water in both respective organic solvents. ${ }^{6}$ These experimental facts are in complete agreement with the findings of the structure of solvation shells of NB and NPOE around both ions that are emphasized in the discussion above.

To conclude, the solvation of the ions by nitrobenzene is taken care of by the molecular polarity $-\mathrm{K}^{+}$interacts with the negative side of the dipole (the nitro group) and $\mathrm{Cl}^{-}$interacts with the positive end (the meta and para aromatic hydrogens). The same holds true for NPOE, because there is very little interaction between the ions and the hydrophobic aliphatic chain, except for an extension of the influence of the $\mathrm{Cl}^{-}$ion to the first hydrogens of the chain.

\section{Conclusions}

In this work, we report a comprehensive MD study on the pure organic solvents nitrobenzene and 2-nitrophenyl octyl ether, paying particular attention to the structure and most important thermodynamic and dynamic properties of both solvents. In terms of structure, the NPOE molecule differs from NB in two fundamental aspects: the conformation of the nitro group and the presence of a long aliphatic chain. Although in the case of $\mathrm{NB}$ the nitro group lays parallel to the benzene ring, it is tilted in the NPOE structure, due to the strong repulsion between the oxygens of the nitro group and the oxygen of the ether bond. The study of various RDFs indeed confirms that the major attractive interactions in both liquids are those between the oxygen atoms of the nitro groups and the aromatic hydrogens. Furthermore, the probability distribution computed for the NPOE aliphatic chain end-to-end distance revealed that this chain is almost fully stretched, with low extent of folding. The calculated thermodynamic properties of both liquids are very similar, and show very good agreement with experimental findings, whenever experimental data are available. 
Regarding the dynamic properties, the viscosity values determined for both NB and NPOE liquids are very close to the experimental data. It is worth noting that the viscosity of NPOE is almost 1 order of magnitude higher than that of nitrobenzene. This results from the specific structure of NPOE, as the spherical aromatic ring prevents the aliphatic chains from sliding past each other, producing a sluggish response to shear. Conversely, the self-diffusion coefficient of NPOE is about an order of magnitude lower than that of NB. Moreover, the ratio between the self-diffusion coefficients of both liquids lies close to the inverse ratio of their viscosities, as suggested by the Stokes-Einstein relation. In addition, we have also studied the solvation and dynamics of the simple $\mathrm{K}^{+}$and $\mathrm{Cl}^{-}$ions in both liquids. It has been found that both ions tend to interact mainly with the oppositely charged polar parts of the aromatic rings of both solvents. This finding is also in accordance with a recent experimental finding, which showed that both NB and NPOE have similar solvation properties toward the ionic solutes. ${ }^{2,11-12}$ Moreover, the calculated theoretical values of the diffusion coefficients of both $\mathrm{K}^{+}$and $\mathrm{Cl}^{-}$ions are in good agreement with the experimentally determined ones in both organic solvents. ${ }^{5-7}$ Note that the values of the diffusion coefficients determined in NPOE are 1 order of magnitude lower than those in NB, following again the Stokes-Einstein relation.

Naturally, NPOE and NB form an interesting pair of solvents for ion transfer studies, because they are identical from the thermodynamic point of view, have similar ion solvation properties but are quite different in terms of dynamic properties. By contrasting the performance of these solvents, one might distinguish between both effects on the ion transfer process. The detailed description of the present NB and NPOE models provides a basis for the study of the interfaces of both organic liquids and water, and we hope to be able to report on that shortly.

Acknowledgment. This work was supported by Fundação para a Ciência e a Tecnologia (FCT), Projects POCTI/QUI/ 41704/2001 and POCTI/QUI/41074/2001. R.G. acknowledges the FCT for the postdoctoral fellowship grant SFRH/BPD/ $14894 / 2004$.

\section{References and Notes}

(1) Marcus, Y. Ion Properties; Marcel-Dekker: New York, 1997.

(2) Scholz, F.; Schroeder, U.; Gulaboski, R. Electrochemistry of Immobilized Particles and Droplets; Springer: Heidelberg, Berlin, 2005.

(3) CRC Handbook of Chemistry and Physics, 78th ed.; Lide, D. R. Ed.; CRC Press: Boca Raton, FL 1997-1998.

(4) Valent, O.; Koryta, J.; Panoch, M. J. Electroanal. Chem. 1987, 226,21
(5) Samec, Z.; Langmaier, J.; Trojanek, A. J. Electroanal. Chem. 1996, $409,1$.

(6) Wilke, S.; Zerihun, T. J. Electroanal. Chem. 2001, 515, 52.

(7) Samec, Z.; Langmaier, J.; Trojanek, A.; Samcova, E.; Malek, J. Anal. Sci. 1998, 14, 35.

(8) Osborne, M. D.; Girault, H. H. Electroanalysis 1995, 7, 714.

(9) Lee, H. J.; Girault, H. H. Anal. Chem. 1998, 70, 4280.

(10) Pereira, C. M.; Tirilly, N.; Martins, M. C.; Silva, F. Fresenius J. Anal. Chem. 2001, 369, 609.

(11) Scholz, F.; Gulaboski, R. ChemPhysChem 2005, 6, 16.

(12) Gulaboski, R.; Galland, A.; Bouchard, G.; Caban, K.; Kretschmer, A.; Carrupt, P.-A.; Girault, H. H.; Scholz, F. J. Phys. Chem. B 2004, 108 , 4565 .

(13) Janssen, R. H. C.; Theodorou, D. N.; Raptis, S.; Papadopoulos, M. G. J. Chem. Phys. 1999, 111, 9711.

(14) Michael, D.; Benjamin, I. J. Electroanal. Chem. 1998, 450, 335.

(15) Schweighofer, K.; Benjamin, I. J. Phys. Chem. 1999, 103, 10274.

(16) dos Santos, D. J. V. A.; Gomes, J. A. N. F. Chem. Phys. Chem. 2002, 3, 946.

(17) Price, M. L. P.; Ostrovsky, D.; Jorgensen, W. L. J. Comput. Chem. 2001, 22, 1340.

(18) Price, D. J.; Brooks, C. L., III. J. Comput. Chem. 2005, 26, 1529.

(19) Shlyapochnikov, V. A.; Khaikin, L. S.; Grikina, O. E.; Bock, C. W.; Vilkov, L. V. J. Mol. Struct. 1994, 326, 1.

(20) Weiner, S. J.; Kollman, P. A.; Nguyen, D. T.; Case, D. A. J. Comput. Chem. 1986, 7, 230

(21) Jorgensen, W. L.; Tirado-Rives, J. J. Am. Chem. Soc. 1988, 110, 1657 .

(22) Brooks, B. R.; Bruccoleri, R. E.; Olafson, B. D.; States, D. J.; Swaminathan, S.; Karplus, M. J. Comput. Chem. 1983, 4, 187.

(23) Berendsen, H. J. C.; van der Spoel, D.; van Drunen, R. Comput. Phys. Comm. 1995, 91, 43.

(24) Lindahl, E.; Hess, B.; van der Spoel, D. J. Mol. Mod. 2001, 7, 306.

(25) Hess, B.; Bekker, H.; Berendsen, H. J. C.; Fraaije, J. G. E. M. J. Comput. Chem. 1997, 18, 1463.

(26) Hockney, R. W.; Goel, S. P. J. J. Comput. Phys. 1974, 14, 148.

(27) Nosé, S. Mol. Phys. 1984, 52, 255.

(28) Hoover, W. G. Phys. Rev. A 1985, 31, 1695.

(29) Parrinello, M.; Rahman, A. J. Appl. Phys. 1981, 52, 7182.

(30) Essman, U.; Perela, L.; Berkowitz, M. L.; Darden, T.; Lee, H.; Pedersen, L. G.J. Chem. Phys. 1995, 103, 8577.

(31) Chandrasekhar, J.; Spellmeyer, D. C.; Jorgensen, W. L. J. Am. Chem. Soc. 1984, 106, 903.

(32) Aqvist, J. J. Phys. Chem. 1990, 94, 8021.

(33) Ciccotti, G.; Jacucci, G.; McDonald, I. R. J. Stat. Phys. 1979, 21 ,

(34) Hess, B. J. Chem. Phys. 2002, 116, 209.

(35) Jorgensen, W. L. Tirado-Rives, J. J. Am. Chem. Soc. 1984, 106 , 6638

(36) Kusano, K.; Wadso, I. Bull. Chem. Soc. Jpn. 1971, 44, 1705.

(37) Almarza, N. G.; Enciso, E.; Bermejo, F. J. J. Chem. Phys. 1992, 96, 4625.

(38) Lee, S. H.; Lee, H.; Pak, H.; Rasaiah, J. C. Bull. Korean Chem. Soc. 1996, 17, 735 .

(39) Jedlovszky, P.; Varga, I.; Gilanyi, T. J. Chem. Phys. 2004, 120, 11839

(40) Hawlicka, E.; Reimschüssel, W. Ber. Bunsen-Ges. Phys. Chem. 1980, 84, 1119.

(41) Yeh, I. C.; Hummer, G. J. Phys. Chem. B 2004, 108, 15873. 